\title{
Beware of the Evidence!: A Cautionary Note
}

\section{Samuel Quek}

Published online: 8 January 2014

(C) Indian Prosthodontic Society 2014

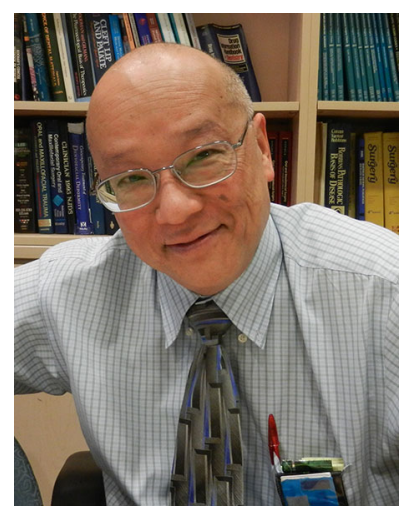

Evidenced-based publications are the "premier" publications that are being read and referred to for valid research information. In this context, it is essential that the reader understands how evidence-based clinical research is conducted. The reader should be aware of research design methodologies, understand the difference between clinical significance and statistical significance, and appreciate the difference between associations and causality. Having this knowledge will allow the cultivation of critical appraisal skills which ultimately benefit the discerning reader of evidence-based studies, enabling them to critically examine the publication in order to determine the validity of the study's research design, and conclusions. Knowledge seekers should never blindly accept the conclusions of evidence-based study just because it was published in a

S. Quek $(\square)$

Rutgers School of Dental Medicine, Newark, NJ, USA

e-mail: queksa@sdm.rutgers.edu peer reviewed journal, by reputable authors from a respected university.

Last year an American Broadcasting Association (ABC) headline took the Dental community by surprise. The headline: "Early Dental X-rays Linked to Brain Tumors" [1]. This ABC report summarized a publication authored by a group of MDs and $\mathrm{PhDs}$ from Yale University School of Medicine's Department of Epidemiology and Public Health, published in the journal of cancer [2]. The article concluded that exposure to traditional dental X-ray investigation over the years may lead to an increase risk of developing meningioma.

A critical review of this publication discovered several flaws in study's methodologies and the conclusions were questioned. An example of one such flawed interpretation was the statement that there was a statistically significant increase in the risk of developing meningioma in patients receiving bitewing $\mathrm{X}$-rays and panoramic $\mathrm{X}$-rays. On the other hand, there was no significant risk of developing meningioma detected in patients receiving full-mouth dental X-rays. Does this make sense? The astute reader would have realized that bitewing X-rays are included in the full mouth series as well, so why are patients at higher risk of developing meningioma in the group that got bitewing X-rays when compared to the full mouth X-ray group. The full report is available from the American Dental Association [3].

Therefore, proficiency in critical review is an essential skill every practitioner who reads journal articles to improve his/her knowledge to better serve his/her patients. The blind acceptance of an author's conclusions without examination of the methodology, analysis and interpretations should be a matter of the pass; as cited even the most reputable authors and journals are susceptible to mistakes at times. 


\section{References}

1. Allen JE (2012) Early dental X-rays linked to brain tumors. http:// abcnews.go.com/Health/GMAHealth/brain-tumors-dental-rays/story? id $=16101200$. Accessed 22 Oct 2013
2. Claus EB et al (2012) Dental X-rays and risk of meningioma. Cancer 118(18):4530-4537

3. Williams J (2012) Experts question X-ray study. http://www.ada. org/news/6979.aspx. Accessed 22 Oct 2013 